\title{
Gravity from the entropy of light
}

\author{
Alessandro Pesci* \\ INFN-Bologna, Via Irnerio 46, I-40126 Bologna, Italy
}

\begin{abstract}
The holographic principle, considered in a semiclassical setting, is shown to have direct consequences on physics at a fundamental level. In particular, a certain relation is pointed out to be the expression of holography in basic thermodynamics.

It is argued moreover that through this relation holography can be recognized to induce gravity, and an expression for the gravitational lensing is obtained in terms of entropy over wavelength of black-body radiation, or, at a deeper level, in terms of maximum entropy over associated space to the elementary bit of information.
\end{abstract}

PACS numbers: 04.60.-m, 05.70.-a, 03.67.-a

\section{INTRODUCTION}

According to the holographic principle [1], only a finite amount of information is allowed to be stored in a region with given bounding area, scaling the limit as the area itself. Its string-theoretical realization was considered in [2].

The original motivation has been very general, since the principle was introduced through combination of gravitational collapse and the basic tenets of quantum mechanics. Somehow, the gravitational context is found to highlight a fundamental redundancy, not visible otherwise, in the quantum-mechanical degrees of freedom used to describe the systems. Through a semiclassical discussion, the aim of this note is to take the reverse path and to spot consequences, if any, on basic physics, once the holographic principle is assumed as primeval starting point.

The most general formulation of the holographic principle at semiclassical level -i.e. with matter degrees of freedom living in a continuous background spacetime- is perhaps the generalized covariant entropy bound [3], which states that the matter entropy $S$ on a terminated lightsheet $L$ is bounded by (in Planck units, the units we will use throughout this note)

$$
S \leq \frac{\Delta A}{4}
$$

where $\Delta A$ is the area difference between the start- and end-surfaces of $L$, and $S$ is calculated through the entropy current $s^{a}$ (assumed to exist), $S=\int_{L} s^{a} \epsilon_{a b c d}$, being $\epsilon_{a b c d}$ the spacetime (which we take 4-dim) 4-volume form. In this work, inequality (11) is assumed to be the precise mathematical formulation of the holographic principle.

We recall a condition for (1) -or a consequence of it when (1) is assumed as starting point- we shall use throughout the paper, which shows up in circumstances in which the effects of spacetime curvature are extremely tiny. The condition, derived and discussed in [4, 5], is as follows : In a spacetime with Einstein's equation, inequality (1) is universally true if and only if a local (i.e. depending on the point) lower-limiting spatial scale $l^{*}$, unrelated to gravity, is assumed to exist in the description of statistical systems, with

$$
l^{*} \equiv \frac{1}{\pi} \frac{s}{\rho+p}=\frac{1}{\pi T}\left(1-\frac{\mu n}{\rho+p}\right)
$$

(where, for the last expression, use of Gibbs-Duhem relation is made). Here $s, \rho, n, p, T, \mu$ are respectively local entropy, mass-energy and number densities, local pressure, temperature and chemical potential (having, this latter, any rest energy included).

For thin plane layers of thickness $l$-actually, that geometric configuration which to the utmost challenges the bound- this becomes

$$
l^{*} \leq l,
$$

*Electronic address: pesci@bo.infn.it 
meaning that below $l^{*}$ the notions themselves of energy, entropy and pressure in the layer become somehow undefined, or, equivalently, that layers thinner than $l^{*}$ cannot be cutted physically if the assigned values of the thermodynamic parameters have to remain unchanged after cutting. The reason why this limiting scale $l^{*}$ should exist can be recognized through consideration of the trivial lightsheets associated with thin plane layers; for them, $\Delta A$ in (1) is, from Raychaudhuri's equation [6], quadratic in $l$, whereas $S$ is (obviously) linear, so that, if a lower limit would not be envisaged for $l$, when $l \rightarrow 0$ inequality (11) would be definitely violated. The bound (1) is attained iff i) we consider plane layers and ii) their thickness just attains the bound (3). $l^{*}$, considered as a time, instead of space, lower-limiting scale (i.e. a lower-limiting time scale in the evolution of statistical systems given by the time it takes light to travel a distance $l^{*}$ ), leads also to foresee [7] the universal bound to the relaxation times [8] of perturbed thermodynamic systems.

As discussed in [9], from a quantum-mechanical standpoint relation (3) can be re-expressed as

$$
l^{*} \leq \lambda
$$

where $\lambda$ is the 'typical' quantum wavelength of constituent particles; a notion which will be sharpened later. What is meant here is that, assuming that quantum mechanics allows for unaltered thermodynamic potentials in physically cutted slices as thin as the $\lambda$ itself of the constituent particles, i) $\lambda$ is the minimum $l$ quantummechanically allowed and ii) (3) leads to (4). For a given system, $\lambda$ in (44) provides the tightest bound coming from holography to that combination of thermodynamic potentials which we denote with $l^{*}$. Only material media with $l^{*}=\lambda$ can attain the bound (11), which is indeed attained when (lightsheets trivially constructed on) plane layers are considered with thickness $l=\lambda$.

\section{A HOLOGRAPHIC LAW IN BASIC THERMODYNAMICS}

In condition (4) any connection with gravity, or curvature, has disappeared. We are left with a flat-spacetime condition, which compares a combination of the thermodynamic potentials of a system with the quantum size of constituent particles; still, this condition is an implication of or a pre-requisite for holography. The aim of this Section is just to emphasize that, due to this, (4) can be read as a sort of basic law of thermodynamics of holographic origin, and discuss its nature.

Let us look, first, at that a well-known basic thermodynamic bound connecting energy $E$, entropy $S$ and size - the circumscribing radius $R$ actually- of a system has already been long since proposed. The Bekenstein universal bound to specific entropy [10]

$$
\frac{S}{E} \leq 2 \pi R
$$

indeed, though originally found through an argument involving black hole physics, was since the beginning recognized as a fundamental thermodynamic bound having nothing to do with gravity.

In [9] the relation of bound (5) with holography has been discussed (see also [11]). At first sight the bounds (5) and the holographic relation (3) seem quite different. One difference is that in (3) $p$ appears also (in $l^{*}$ ).

As this regard we point out that, considering the conditions of the original argument bringing to (5) through the use of the generalized second law [12, 13], in circumstances more general than those originally considered a contribution from the work done by pressure should also be present.

The basic fact used in the derivation of (5) is, indeed, that if a body with energy $E$ and circumscribing radius $R$ (and negligible self-gravity) is swallowed by a black hole, a lower limit definitely exists to the increase of surface area of the hole, given by $8 \pi E R$ [12, 13]. Then, from this, and imagining that given a body a process can always be found for which this limit is attained, through the use of the generalized second law for such a process (5) is obtained.

Now, if we consider for simplicity a static black hole and, instead of assuming that a whole body is swallowed, we dump in, just when it is at its first contact with the horizon, a small element of proper thickness $l$ and crosssectional area $A$ of an indefinitely extended fluid (i.e. if, contrary to [12, 13], we no longer require the stress tensor $T_{a b}$ to be vanishing outside the body), for a perfect fluid assumed momentarily at rest in the local static frame of the metric to first order in $T_{a b}(\mathrm{cf}$. [14]) we get

$$
\Delta M=\int_{0}^{l} d l^{\prime} \int d^{2} S T_{a b} \xi^{a} k^{b}=\kappa \int_{0}^{l} d l^{\prime} A l^{\prime}(\rho+p)=\kappa A(\rho+p) \frac{l^{2}}{2}=\kappa \frac{l}{2}(E+p V),
$$

where $V$ is the proper volume of the fluid element, $\kappa$ is the surface gravity of the hole, $l^{\prime}$, the proper length in the fluid local frame, is the chosen affine parameter for the null geodesics (with tangent $k^{a}$ ) on the horizon, $d^{2} S$ 
is the cross-section element of the horizon at $l^{\prime}$ and $\xi^{a}$ is the Killing field, orthogonal to the horizon, which is timelike at infinity. In (6), use of the relation $\kappa l^{\prime} k^{a}=\xi^{a}$ with $\kappa=$ const is made, which is appropriate in so far as the change in the black hole geometry in the process can be neglected, which always is the case provided we choose $M$ large enough. Here we are also assuming $V$ small enough to allow for thermodynamic potentials approximately constant in it.

From (6) we get the minimum horizon area increase, which is $\frac{8 \pi}{\kappa} \Delta M=4 \pi l(E+p V)$, from which, through use of the generalized second law, we obtain

$$
\frac{S}{E+p V} \leq \pi l .
$$

So, if the element we drop in is, say, a gas contained in a box, and if the thermodynamic system we are considering consists of both the gas and the constraining walls, we are led to $S / E \leq \pi l$ (as in the derivation in [13]), ${ }^{1}$ if instead our system consists of the gas alone, our argument says that the fundamental bound should be given by expression (7), i.e. with the term $p V$.

Inequality (7) manifestly coincides with condition (3). In [9], it has been shown that, even if we start from (7), for macroscopic bodies we are lead anyway to (5) (for whichever strength, indeed, of the gravitational effects).

Still, the bound expressed by condition (4) strongly differs from the Bekenstein bound, being the former actually enormously tighter than the latter. For a spherical homogeneous system with radius $R$, for example, the Bekenstein bound says that the ratio $S / E$ is bounded by something orders of $R$, while according to (4) this same ratio is bounded by orders of $\lambda$, the 'typical' wavelength of constituent particles, with always $\lambda \leq R$, and in general $\lambda \ll R$. Thus, holography, which, too, has among its consequences the Bekenstein bound, can be seen to imply for basic thermodynamics a bound, condition (4), in general extremely stronger than Bekenstein's one. Bound (4) seems thus could be considered as the fundamental requirement in basic thermodynamics of the whole gravity-thermodynamics connection. It is the basic-thermodynamic imprint of holography.

Let us give a closer look to bound (4). In the transition from (3) to (4), the particle 'typical' wavelength $\lambda$ is, we said, a sort of minimum thickness below which, in view of the uncertainty relations, the value of thermodynamic potentials in a physically-cutted slice are found different than before cutting. It can be defined more precisely considering that a limiting thickness $l_{\text {min }}$ should exist for which the quantum spread in momentum $\Delta p_{x}^{i n d}$, induced by constraining the particles in the given thickness, becomes equal to the intrinsic momentum spread $\Delta p_{x}^{\text {int }}$ (dictated by the assigned thermodynamic conditions)

$$
\Delta p_{x}^{i n d}=\Delta p_{x}^{i n t}
$$

(here $x$ labels the direction orthogonal to the slice). For constituent particles all with a same quantum spatial uncertainty which still we denote $\lambda$, condition (8) will be reached by definition just when $l_{\text {min }}=\lambda$. In the general case we define $\lambda$ as that thickness which gives (8). For a Boltzmann gas the value of a so-defined $\lambda$ is close to thermal de Broglie wavelength.

When checked on actual systems, condition (4) is found in general satisfied by far [5]. For the most entropic systems it appears, instead, practically attained. For black body radiation - and in general for ultrarelativistic constituent particles-, for example, an argument described in [5] suggests

$$
\lambda=1 / \pi T
$$

and thus $l^{*}=\frac{1}{\pi T}\left(1-\frac{\mu n}{\rho+p}\right)=\lambda$, being $\mu=0$. This prompts to consider the uncertainty relations as the mechanism which leads to (4), i.e. to (3).

From this perspective, condition (44) (and, thus, (3)) appears to be a basic thermodynamic relation arising from quantum mechanics alone. This relation, when combined with Einstein's lensing, leads inexorably to the generalized covariant bound to entropy, that is to holography, and vice versa is the unavoidable consequence of the latter in basic thermodynamics.

As known, the generalized second law follows from the generalized entropy bound, assuming the validity of the ordinary second law [3]. From the above this means that, besides ordinary second law, just another ingredient from basic thermodynamics is needed, and is enough, to give in a gravitational context the generalized

\footnotetext{
${ }^{1}$ We are grateful to R. Bousso for correspondence on this point.
} 
second law: relation (44). We see that this relation turns out to be that component of basic thermodynamics responsible for the 'generalized part' of the generalized second law, i.e. that part which deals with processes involving horizons.

If we imagine to start from the holographic principle without any notion of quantum mechanics, bound (1) (which, consistently, should thus be understood as the log of the number of allowed different microscopic configurations (instead of the $\log$ of the number of allowed orthogonal quantum states)) requires, we have seen, the existence of a lower-limiting scale $l^{*}$. The existence of this spatial limit would suggest that a 'size' should be assigned to the elementary constituents of matter, while the value of the spatial limit (expression (2)) would imply that, at least for ultrarelativistic systems, this 'size' of the constituents should be related to their momentum by the uncertainty relations. That is, holography demands for a microscopic description of matter, discrete in itself anyhow if finite values are to be assigned to the entropy of generic systems, which too is driven by what we know as the uncertainty relations, i.e. it somehow demands for quantum mechanics. ${ }^{2}$

\section{A STATISTICAL ORIGIN FOR GRAVITY}

At the end, what we have seen so far is that, if the bundles of light rays actually shrink gravitationally, the lower-limiting length $l^{*}$, which manifests quantum mechanics, is the effect of bound (1). But, what about the source of this shrinking? Looking at (1), we see that holography demands that, even in absence of interactions of any kind, some mechanism must be at work which shrinks the bundles of light rays when going through matter. The mere existence of some entropy in a region requires, there, a focusing. Bound (11) moreover knows nothing else than entropy and focusing, so that it is quite natural, as far as we take (1) as our primeval starting point, to somehow suspect entropy as the source of focusing.

Massive bodies can be considered for which the entropy, when temperature is near absolute zero, can be negligibly small, but the focusing sizeable. So, if entropy is to be responsible of focusing, which entropy should we take ? It is clear that if, for assigned $\rho, p$ and $\lambda$, the rate of shrinking could be determined by a value of $s$ as high as the limit $\frac{s}{\rho+p}=\pi \lambda$ in (4), any matter would comply with the bound (1). Thus, the rate of shrinking could be set by the request that bound (1) be always satisfied, and exactly attained by the most entropic systems.

The perspective we advocate here is that in giving some piece of matter what we are really doing is to allocate some maximum amount of information or entropy, let us call it 'intrinsic' information/entropy, associated with it. The bound then says that this 'intrinsic' information/entropy must focus light rays at a precise rate.

We can derive the value of this rate through consideration of material systems which do attain the limit in (4). For the terminated lightsheet of a plane layer of a photon gas with thickness $l$ as small as the limit $l=l_{\min }=\lambda=1 / \pi T$, entropy just attains the limit in (1), as well as in (3) and (4). This means that at these conditions the shrinking of the null congruence traversing the layer is given by

$$
-\Delta A=4 S=4 s A \lambda=4 \pi(\rho+p) A \lambda^{2}=4 \pi A \lambda^{2} T_{a b} k^{a} k^{b}
$$

where $T_{a b}$ is the stress energy of the photon gas and $k^{a}$ are the tangents to the null congruence with respect to the parametrization given by $l$, and use of (4) has been made. On the other hand, from geometry the shrinking is connected, through Raychaudhuri's equation, to the Ricci tensor $R_{a b}$. The Raychaudhuri equation, in our circumstances of vanishing shear and initially vanishing expansion $\theta=\frac{1}{A} \frac{d A}{d l}$, for very small $l$ reads

$$
\frac{d \theta}{d l}=-R_{a b} k^{a} k^{b}=\text { const }
$$

from which we get $\theta=-l R_{a b} k^{a} k^{b}$ and thus the mentioned quadratic dependence of $\Delta A$ on $l$ has the form

$$
-\Delta A=-\int_{0}^{l} \theta A d l^{\prime}=A \frac{l^{2}}{2} R_{a b} k^{a} k^{b} .
$$

From equation (10) this gives

$$
R_{a b} k^{a} k^{b}=8 \pi T_{a b} k^{a} k^{b} .
$$

${ }^{2}$ Cf. [15]; see also [7, 9]. 
This is not a surprise. The lensing turns out to be just that given by Einstein's equation. As it must be, since bound (11) has Einstein's lensing built-in. The real point here is the perspective: the focusing is determined by ('intrinsic') information/entropy through holography (bound (1)). That is to say, starting from holography without any notion of gravity and knowing only of information/entropy, we end up with what we call gravity, and this points to a direction akin to [16, 17] (last Section of both) and [18], to some extent. ${ }^{3}$

In [21], a thermodynamic interpretation of Einstein's equation (in which this reveals itself as an equation of state) has already been given from an assumed proportionality of horizon entropy and area. The present attempt is supposed to provide a step forward in that, through consideration of information as fundamental, the existence and strength of what we call gravity is reduced to a principle of maximum allowed amount of information inside any closed surface. That is, what really matters here is not just the thermodynamic nature of Einstein's equation (a point, this, of paramount importance indeed, as for its implications on the opportunity of any attempt to quantize this equation as well as for its accounting of the occurrence of thermodynamic laws for classical black holes [21]), but that the occurrence itself of gravity is understood as what must happen in order that a certain primeval property of entropy be preserved.

Looking at (10), we see that the role played by $\pi(\rho+p)$ in determining the shrinking of the congruence by the photon gas, can be viewed as played by $s / \lambda$. This suggests that, given some matter, it is the 'intrinsic' entropy density over wavelength, namely the maximum entropy density over wavelength at the given energy density+pressure, what should be considered as the proper source of focusing, and what determines the gravitational acceleration.

Considering light rays traversing orthogonally a thin plane layer of matter, using the focusing equation [22], which, since the shear is vanishing, reads

$$
\frac{d^{2} A^{1 / 2}}{d l^{2}}=-\frac{1}{2} R_{a b} k^{a} k^{b} A^{1 / 2}
$$

we have that, assuming rotational symmetry around the propagation axis (as it is the case if local matter is assumed to be the only source of the field), the local-frame acceleration $a_{t}$ felt by the photons of the congruence while going through matter being a distance $d$ apart can be expressed as

$$
a_{t}=-\frac{1}{2} R_{a b} k^{a} k^{b} d=-4 \frac{s}{\lambda} d
$$

denoting with $s / \lambda$ the 'intrinsic' entropy density per unit wavelength. This same expression can be used to determine also the local acceleration with respect to the origin, taken halfway between the photons, if $d$ changes its meaning becoming the distance from the origin.

Expression (15) fixes the gravitational acceleration felt by photons in the local frame, due to the presence of local matter, as determined by its 'intrinsic' entropy. The operational meaning is that given a material medium with some local values $\rho_{m}$ and $p_{m}$ of energy density and pressure, the local matter affects through holography the motion of photons in the way expressed by (15) (and this effect is what we call gravity), where $s / \lambda$ is the entropy density per unit wavelength of a photon gas having energy density $\rho=\frac{3}{4}\left(\rho_{m}+p_{m}\right)$ and $\lambda=1 / \pi T$, where $T$ is its black body temperature. The acceleration in (15) is thus expressed in terms of entropy density per unit wavelength of that black body radiation which gives the 'intrinsic' informational content of the local matter we are considering.

\section{CONCLUDING REMARKS}

In conclusion, what we have tried to show in the paper is that the holographic principle, assumed as primeval starting point, implies both a basic relation in flat-spacetime thermodynamics, relation (4) (argued to be more fundamental than the Bekenstein bound), and the curvature effects we call gravity, with a new entropy -different from actual thermodynamic entropy-, the 'intrinsic' entropy (per $\lambda$ ) of a body, playing the role of source of the curvature. An expression of the gravitational acceleration in terms of it has also been given (relation (15)).

We can summarize what we have seen as follows. The Einstein's focusing we have obtained, or the explicit expression for the gravitational acceleration in equation (15), permits to view what we call 'gravitational effects'

\footnotetext{
${ }^{3}$ We notice that in [19] another derivation of gravity is given, independent, like the present attempt, of [16-18]. In it, gravity is derived from the Laundauer principle in quantum information theory as applied to horizons. In [20], moreover, the EinsteinHilbert action, or more general actions, are derived starting from black hole entropy as calculated within loop quantum gravity.
} 
as actually holography at work, and the 'gravitational' acceleration as a 'holographic' acceleration. Gravity is merely all what is needed for the 'holographic' property of entropy to be preserved. In particular in equation (15) we can read directly the strength of a gravitational acceleration per given amount of 'intrinsic' information associated with matter.

Relation (4) (with expression (2)), in the form of uncertainty-like relations, establishes the rule for finding the 'intrinsic' information allocated with the assigned matter (so that the meaning of the uncertainty relations would be in their being what provides the informational content of matter). This information (per $\lambda$ ) is defined as the maximum entropy per $\lambda$ we can associate to that matter, i.e. the value which just attains (44); and it turns out to be the entropy per $\lambda$ of 'equivalent' (in a definite sense) black body radiation. This choice is dictated by the request that the strength of the holographic focusing be just that needed for the entropy in a (terminated) lightsheet to be universally bounded (i.e. for every lightsheet geometry) by the number $\Delta A / 4$, and just attained for the most challenging geometric choices. Thus, holography, by saying that the number of allowed degrees of freedom inside a given closed surface is bounded (by a value proportional to the area of the boundary), induces curvature effects determined by the 'intrinsic' degrees of freedom carried by matter (effects with a strength depending on the value of the bound), and this constitutes what we call gravity.

To speak of entropy per wavelength of black body radiation means to speak of entropy per 1-bit-ofinformation thickness, since the single bits of information are carried by the single constituent photons and we have $\simeq 1$ photon every $\lambda^{3}$ volume. This suggests a deeper description of what we have discussed. Indeed, holography can be stated to imply that the allowed number of elementary bits of information in a layer of 1 bit thickness at given sum of energy and pressure energy in the layer is bounded. To the extent that concepts like bit of information and energy and pressure energy of a bit can be regarded as primeval and, as such, meaningful even in absence of space, holography is pre-existing to space (cf. [16 18], and [23]). In this perspective, when space is introduced as the information on 'where' information is, the energy in the bit should spread to keep unchanged the elementary amount of information for the bit, and this would be quantum mechanics. When expressed in terms of this notion of space, holography would then become the metric theory which describes gravity.

I am grateful to Alessio Orlandi for fruitful discussions on some of the arguments considered in the note.

[1] G. 't Hooft, "Dimensional reduction in quantum gravity", essay dedicated to Abdus Salam, published in Salamfest (1993) 0284, gr-qc/9310026.

[2] L. Susskind, "The world as a hologram", J. Math. Phys. 36 (1995) 6377, hep-th/9409089.

[3] É.É. Flanagan, D. Marolf and R.M. Wald, "Proof of classical versions of the Bousso entropy bound and of the generalized second law", Phys. Rev. D 62 (2000) 084035, hep-th/9908070.

[4] A. Pesci, "From Unruh temperature to the generalized Bousso bound", Class. Quantum Grav. 24 (2007) 6219, arXiv:0708.3729.

[5] A. Pesci, "On the statistical-mechanical meaning of the Bousso bound", Class. Quantum Grav. 25 (2008) 125005, arXiv:0803.2642.

[6] R.M. Wald, General Relativity (The University of Chicago Press, Chicago, 1984).

[7] A. Pesci, "A note on the connection between the universal relaxation bound and the covariant entropy bound", Int. J. Mod. Phys. D 18 (2009) 831, arXiv:0807.0300.

[8] S. Hod, "Universal bound on dynamical relaxation times and black-hole quasinormal ringing", Phys. Rev. D 75 (2007) 064013, gr-qc/0611004.

[9] A. Pesci, "A proof of the Bekenstein bound for any strength of gravity through holography", Class. Quantum Grav. 27 (2010) 165006, arXiv:0903.0319.

[10] J.D. Bekenstein, "Universal upper bound on the entropy-to-energy ratio for bounded systems", Phys. Rev. D 23 (1981) 287.

[11] R. Bousso, "Light-sheets and Bekenstein's bound", Phys. Rev. Lett. 90 (2003) 121302, hep-th/0210295.

[12] J.D. Bekenstein, "Black holes and entropy", Phys. Rev. D 7 (1973) 2333.

[13] J.D. Bekenstein, "Generalized second law of thermodynamics in black-hole physics", Phys. Rev. D 9 (1974) 3292.

[14] R.M. Wald, Quantum field theory in curved spacetime and black hole thermodynamics (The University of Chicago Press, Chicago, 1994).

[15] R. Bousso, "Flat space physics from holography", JHEP05(2004) 050, hep-th/0402058

[16] T. Padmanabhan, "Thermodynamical Aspects of Gravity: New insights", Rept. Prog. Phys. 73 (2010) 046901, arXiv:0911.5004.

[17] T. Padmanabhan, "Equipartition of energy in the horizon degrees of freedom and the emergence of gravity", Mod. Phys. Lett. A 25 (2010) 1129, arXiv:0912.3165.

[18] E.P. Verlinde, "On the origin of gravity and the laws of Newton", arXiv:1001.0785.

[19] J.-W. Lee, H.-C. Kim and J. Lee, "Gravity from quantum information", arXiv:1001.5445.

[20] F. Caravelli and L. Modesto, "Holographic actions from black hole entropy", arXiv:1001.4364. 
[21] T. Jacobson, "Thermodynamics of spacetime: the Einstein equation of state", Phys. Rev. Lett. 75 (1995) 1260, gr-qc/9504004

[22] C.W. Misner, K.S. Thorne and J.A. Wheeler, Gravitation (W.H. Freeman and Company, New York, 1973).

[23] F. Markopoulou and L. Smolin, "Holography in a quantum spacetime", hep-th/9910146. 